\title{
Polarized Optical Scattering Signatures from Biological Materials
}

\author{
W.E.Martin ${ }^{a}{ }^{\star}$, E. Hesse ${ }^{b}$, J.H.Hough ${ }^{a}$, W.B. Sparks ${ }^{c}$, C.S. Cockell ${ }^{d}$, Z. Ulanowski ${ }^{b}$, T. \\ A. Germer ${ }^{\mathrm{e}}$, P.H. Kaye \\ ${ }^{a}$ Centre for Astrophysics Research, University of Hertfordshire, UK \\ ${ }^{b}$ Science and Technology Research Institute, University of Hertfordshire, UK \\ ${ }^{c}$ Space Telescope Science Institute, Baltimore, USA \\ ${ }^{d}$ Geomicrobiology Research Group, Open University, UK \\ ${ }^{e}$ National Institute of Standards and Technology, USA \\ ${ }^{*}$ Corresponding author. Tel.:+44 1707285263 \\ E-Mail address: w.e.martin@herts.ac.uk (W.E. Martin)
}

\section{Abstract}

The polarization of laser light backscattered from biological samples has been measured over the wavelength range 350 to $850 \mathrm{~nm}$. Incident circular, linearly polarized, and unpolarized light produces significant spectral features in the case of samples containing chlorophyll such as leaves and cyanobacteria. Polarization scattering signatures are observed around the chlorophyll 'red edge' that may have diagnostic value in the search for life signatures from extrasolar planets.

\section{Introduction}

We describe polarimetry measurements on biological materials, leaves and natural microbial biofilms, and inorganic samples at the Polarimetry Laboratory of the University of Hertfordshire. The emphasis is on a parametric survey at visible wavelengths of light scattering from naturally occurring samples containing photoactive organic structures. The experiments form part of an ongoing project to establish a data base of potential optical polarization signatures for the detection of extrasolar life. The search for techniques to identify significant optical signatures of biological activity on exoplanets has been discussed in several papers [1-13, 43, 44].

There have been a number of astrobiology studies focused on the 'vegetation red edge', the optical signatures arising from the very sharp chlorophyll absorption at around 680nm [7-13]. The rapid increase in light scattered from green plants at this wavelength is well known. Less studied is the scattering behaviour in polarized light near the absorption bands (the Cotton effect), particularly circular polarization. Such scattering produces differences in right and left-handed circular polarization [14,15]. Sparks et al $[5,6]$ showed this behaviour measuring the scattering of unpolarized light from cultured cyanobacteria and leaves. Our measurements on scattered light, using a tunable laser, show significant polarization with incident circular and linear polarized light and unpolarized light on a wide range of plant materials and microorganisms.

The emphasis in our work and that of Sparks is on circular polarimetry because of the possibility of detecting the unique 'handedness' associated with the chiral nature of biologically active material. This may aid the detection of life through the identification of polarization scattering signatures in addition to purely spectroscopic means, i.e., the presence of free atmospheric oxygen [16]. 


\section{Polarimeters and the Mueller Matrix}

Recent spectropolarimeter designs for astronomy $[17,18]$ have employed photoelastic modulators (PEM) at the heart of full or partial Stokes parameter measurements. Our laboratory-based design follows closely the experience with the PlanetPol [17] polarimeter. Figure 1 shows the general layout.

The light source for the measurements is a tunable, modelocked, Ti:Sapphire laser followed by second harmonic generating (SHG) crystals and/or an optical parametric oscillator (OPO). The laser (Mai Tai HP, Newport-Spectra) can produce 100fs pulses but we only use the quasi-continuous wave (CW) mode-locked pulse train for the elastic scattering measurements described here. The spectral region from $345 \mathrm{~nm}$ to $1360 \mathrm{~nm}$ is covered by the laser system and OPO (Coherent-Mira Basic) with gaps at $510 \mathrm{~nm}$ to $550 \mathrm{~nm}$ and $1020 \mathrm{~nm}$ to $1100 \mathrm{~nm}$. The laser system wavelength could be selected to $\pm 1 \mathrm{~nm}$ and linewidths varied between $2-5 \mathrm{~nm}$ over the spectral range monitored by a spectrometer (Ocean Optics HR4000).

The average power to the sample is kept small $(<0.01 \mathrm{~mW})$ and measurement time is kept short to avoid heating and damage to the biological samples. At even these low power densities $\left(\sim<0.2 \mathrm{~mW} \mathrm{~cm}^{-2}\right)$ the leaf samples in particular exhibited changes in scattering during the first few seconds of illumination that were not permanent. This is well known light exposure time behaviour $[19,20]$. The measurements we report here are the scattering values achieved after more than $\sim 2 \mathrm{sec}$ illumination and in no case was any permanent damage to the sample evident. The variability of biological samples is a common problem and we configured the apparatus for consistent sample placement and orientation.

The polarimeter is configured to measure elements of the Stokes scattering matrix with an emphasis on high sensitivity circular polarization backscattering measurements. The incident laser beam is attenuated with neutral density filters and passes through a Glan-laser prism to define the input polarisation. A bare surface reflection from a BK-7 glass wedge is used to monitor the input average power to the sample. A pair of apertures in front of the sample and in front of the power sensor are arranged to be at conjugate points so that the incident laser beam is monitored by the power sensor. Protected silver mirrors are used throughout because of the very wide wavelength range. The beam height is constant throughout the optical path to avoid polarization changes at the metallic mirrors. There are no reflectors or optics other than the final waveplates in the beam path from the defining polarizer to the sample.

For the defined-input polarization $(\mathrm{PI})$ experiments the vertically polarized laser beam is passed through a super-achromatic [310nm-1100nm] quarter wave retarder [B. Halle] held in a precision rotator. By rotating the crystal axis of the retarder from 0 deg to $\pm 45 \mathrm{deg}$ with respect to the laser beam polarization we can obtain linear vertical (s) and left and right circular polarization incident on the sample. For the time-averaged-unpolarized (TAU) incident light measurements we replace the quarter wave retarder with a matching half wave retarder that could be rotated at up to $300 \mathrm{rpm}$ to provide unpolarized incident light [21]. 
We chose a fixed incidence and scattering angle for these measurements which are primarily aimed at determining the spectral dependence of polarization in scattered light thereby extending the many previous multiple angle, fixed wavelength studies. The sample is held such that the angle of incidence on the sample is 45deg and the light scattered is measured at a fixed angle of 135deg, i.e., backscattered from the sample surface. This configuration avoids the incident beam specular (Fresnel) reflection from flat samples. Reflections from rough or non-flat surfaces can add significant background to the scattering from subsurface features. (A single reflection of an incident unpolarized beam from a dielectric surface of $n=1.4$ at an incidence angle of $22.5 \mathrm{deg}$ is $23 \%$ (s) polarized.) Pure surface (Fresnel) reflections can be eliminated by techniques such as index-matching, but these were not used here. Many of our samples show large scattering that is position dependent because of surface roughness.

The analyzer for the light scattered from the sample uses a rotatable superachromatic half wave plate followed by the photoelastic modulator [PEM] (Hinds I/FS20) and a Wollaston prism polarizer. The vertical and horizontal polarized light is then detected by photomultiplier modules [Hamamatsu H6779-01] and the resulting signals measured with a lock-in amplifier (SRS SR830) and a digital oscilloscope (Tek TPS2024) programmed as an averaging voltmeter. The solid angle of the light collection is $0.035 \mathrm{rad}$ set by the $8 \mathrm{~mm}$ PMT apertures. The samples have a variety of geometries and the relatively small incident beam diameter $(3-4 \mathrm{~mm}$, the unexpanded output diameter of the laser system) means that sample to sample variations can be significant. The experimental configuration is sensitive to small angle deviations with smooth samples e.g. those that are similar to a Fresnel reflection from a pure dielectric. We see variations of $\sim 10 \%$ in total scattering measurements on different samples from the same source, e.g. leaves. With fixed illumination on a given sample we can achieve repeatable measurements within $\sim 2 \%$ over the entire spectrum with smaller variations at fixed wavelengths.

The PI and TAU experimental configurations do not measure the complete Stokes scattering coefficients $[22,23]$ as not all input polarizations are implemented; $(p)$ and \pm 45 deg linear polarizations are omitted so only 10 of the 16 coefficients can be uniquely determined. The analyzer can measure all Stokes components of the scattered light. The experimental instruments and data handling were controlled through a LabView program that automated much of the data taking. Each data point is the average of five sequential measurements of amplitude and phase. The standard deviation is calculated for the sample population and is displayed as plus and minus 1 std where shown.

\section{Stokes Parameters and Calibration}

The conventional Mueller-Stokes formulation of light scattering from a surface can be expressed as

$S^{\prime}=M S$

Where $S$, the input light polarisation state is given by $[I, Q, U, V]^{\top}$ and $M$ is the $4 \times 4$ scattering matrix 


$$
M=\left|\begin{array}{llll}
\mathrm{m}_{11} & \mathrm{~m}_{12} & \mathrm{~m}_{13} & \mathrm{~m}_{14} \\
\mathrm{~m}_{21} & \mathrm{~m}_{22} & \mathrm{~m}_{23} & \mathrm{~m}_{24} \\
\mathrm{~m}_{31} & \mathrm{~m}_{32} & \mathrm{~m}_{33} & \mathrm{~m}_{34} \\
\mathrm{~m}_{41} & \mathrm{~m}_{42} & \mathrm{~m}_{43} & \mathrm{~m}_{44}
\end{array}\right|
$$

The scattering coefficients $m_{i j}$ are dependent on wavelength, incident and scattered angles for the experimental configuration. For our measurements the incidence and scattered angles are fixed as described previously.

Passing S' through an analyzer A gives

$S^{\prime \prime}=A S^{\prime}=A M S=\left[I^{\prime \prime}, Q^{\prime \prime}, U^{\prime \prime}, V^{\prime \prime}\right]^{\top}$

For the analyzer of Fig 1 with a setting of 0 deg for the half-wave plate, the Mueller matrix $A$ is given by

$A_{p}=1 / 2\left|\begin{array}{cccc}1 & c & 0 & -s \\ 1 & c & 0 & -s \\ 0 & 0 & 0 & 0 \\ 0 & 0 & 0 & 0\end{array}\right|, A_{s}=1 / 2\left|\begin{array}{cccc}1 & -c & 0 & s \\ -1 & c & 0 & -s \\ 0 & 0 & 0 & 0 \\ 0 & 0 & 0 & 0\end{array}\right|$

through the Wollaston prism. $\quad c=\cos \delta, s=\sin \delta, \delta$ is the retardance phase shift introduced by the PEM, and the subscripts $A_{x}$ refer to the two orthogonal linear outputs of the prism. For a setting of $22.5 \mathrm{deg}$ for the half wave plate we have

$A_{p}=1 / 2\left|\begin{array}{cccc}1 & 0 & c & -s \\ 1 & 0 & c & -s \\ 0 & 0 & 0 & 0 \\ 0 & 0 & 0 & 0\end{array}\right|, A_{S}=1 / 2\left|\begin{array}{cccc}1 & 0 & -c & s \\ -1 & 0 & c & -s \\ 0 & 0 & 0 & 0 \\ 0 & 0 & 0 & 0\end{array}\right|$

Carrying through the matrix multiplication for the (s) polarization channel we find that the light intensity on the detector is

$I^{\prime \prime}=1 / 2\left(l^{\prime}-c Q^{\prime}+s V^{\prime}\right)$ for 0 deg half-wave plate orientation and

$l^{\prime \prime}=1 / 2\left(l^{\prime}-c U^{\prime}+s V^{\prime}\right)$ for 22.5 deg half-wave plate orientation.

For a sinusoidal retardance modulation from the PEM, $\delta=B \sin \omega t$ with $\mathrm{B}$ the modulation amplitude and $\omega$ the modulation frequency. Expanding $c$ and $s$ using the Bessel functions $\mathrm{J}_{\mathrm{n}}(\mathrm{x})$ and collecting the magnitude of the terms in multiples of $\mathrm{k} \omega \mathrm{t}$ we have the following Table 1

\begin{tabular}{|c|c|l|l|}
\hline k & l' & \multicolumn{1}{|c|}{0 deg } & \multicolumn{1}{|c|}{22.5 deg } \\
\hline 0 & $\mathrm{dc}$ & $1 / 2\left(\mathrm{I}^{\prime}-\mathrm{Q}^{\prime} \mathrm{J}_{0}(\mathrm{~B})\right)$ & $1 / 2\left(\mathrm{I}^{\prime}-\mathrm{U}^{\prime} \mathrm{J}_{0}(\mathrm{~B})\right)$ \\
\hline 1 & $\omega \mathrm{t}$ & $\mathrm{V}^{\prime} \mathrm{J}_{1}(\mathrm{~B}) \sin \omega \mathrm{t}$ & $\mathrm{V}^{\prime} \mathrm{J}_{1}(\mathrm{~B}) \sin \omega t$ \\
\hline 2 & $2 \omega t$ & $\mathrm{Q}^{\prime} \mathrm{J}_{2}(\mathrm{~B}) \cos 2 \omega t$ & $\mathrm{U}^{\prime} \mathrm{J}_{2}(\mathrm{~B}) \cos 2 \omega t$ \\
\hline 3 & $3 \omega t$ & $\mathrm{~V}^{\prime} \mathrm{J}_{3}(\mathrm{~B}) \sin 3 \omega t$ & $\mathrm{~V}^{\prime} \mathrm{J}_{3}(\mathrm{~B}) \sin 3 \omega t$ \\
\hline
\end{tabular}

Table 1. Magnitude of Stokes components as a function of modulation frequency and half-wave plate settings. 
plus higher order terms. Normally terms of $k=3$ and above are ignored because of the decreasing size of the $J_{n}(x)$ coefficients. By selecting $B=2.4048$ radians, $J_{0}(B)=0$ and we can make the $Q$ ' and $U$ ' dependent dc terms go to zero. Note that $J_{3}(2.4048)=0.199$ so that there will be significant modulation at $3 \omega$ with our choice of B.

The experimental values given in this paper can be expressed as the normalised polarization fractions

$Q=l^{\prime \prime}(2 \omega t) / l^{\prime \prime}(d c)=Q^{\prime} J_{2}(B) \cos 2 \omega t / l^{\prime \prime}(d c), 0 d e g$

$U=l^{\prime \prime}(2 \omega t) / l^{\prime \prime}(d c)=U^{\prime} J_{2}(B) \cos 2 \omega t / l '(d c), 22.5 \mathrm{deg}$

$V=l^{\prime \prime}(\omega t) / I^{\prime \prime}(d c)=V^{\prime} J_{1}(B) \sin \omega t / l "(d c), 0$ deg

$\mathrm{Q}, \mathrm{U}$, and $\mathrm{V}$ are related to the degree of polarization $\mathrm{p}$ by

$0 \leq p=\sqrt{Q^{2}+U^{2}+V^{2}} \leq 1$,

and $\mathrm{Q}, \mathrm{U}$, and $\mathrm{V}$ are fractional values between 0 and 1. Pure circular polarization, for example, can be expressed as $\mathrm{p}=\mathrm{V}=1$.

Relating the Mueller scattering coefficients to the Stokes vectors of the scattered light we have for $S^{\prime}$ respectively for unpolarized light inputs $[1,0,0,0]^{\top},(\mathrm{s})$ inputs $[1,-$ $1,0,0]^{\top}$ and right and left circular inputs $[1,0,0, \pm 1]^{\top}$

$S^{\prime}=\left|\begin{array}{l}I^{\prime} \\ Q^{\prime} \\ U^{\prime} \\ V^{\prime}\end{array}\right|=\left|\begin{array}{l}m_{11} \\ m_{21} \\ m_{31} \\ m_{41}\end{array}\right|, \quad\left|\begin{array}{l}m_{11}-m_{12} \\ m_{21}-m_{22} \\ m_{31}-m_{32} \\ m_{41}-m_{42}\end{array}\right|, \quad\left|\begin{array}{l}m_{11} \pm m_{14} \\ m_{21} \pm m_{24} \\ m_{31} \pm m_{34} \\ m_{41} \pm m_{44}\end{array}\right|$

To simplify the notation in the presentation of the results for the PI measurements a compact notation is used in the figures as in the following examples

$V(s)=m_{41}-m_{42}, Q(R)=m_{21}+m_{24}, V(L)=m_{41}-m_{44}, U(L)=m_{31}-m_{34}$, etc.

These are respectively the Stokes polarization fractions measured with the incident polarization given in parentheses

The scattering coefficients are obtained from the $Q, U, V$ measurements directly. Individual scattering coefficients can be calculated by combining the measurements on a given sample. Note that the normalization to the dc level is equivalent to setting $m_{11}=1$ and the resulting normalized scattering coefficients are presented in the results.

The calibration of the polarimeter was performed using reflections from bare fused silica prisms and unprotected aluminium mirrors at an incidence angle of 22.5deg. Circular and linear responses were measured for all input polarizations incident on the reflectors [24] and were within $2 \%$ of the theoretically calculated values for the 
reflections. The smallest $\mathrm{Q}, \mathrm{U}, \mathrm{V}$ polarization fractions that could be detected with small standard errors using five sample measurements was .0002.

Scattering from Spectralon standards [25] was measured periodically and used to determine the system spectral response for the total scattering and to check the sensitivity of the polarimeter with a sample producing low polarization in scattered light. The lack of an agreed standard for low polarization scattering is an issue that is partly addressed here by the use of this uncalibrated but widely available standard material.

The optical compromises associated with operating over the large wavelength range meant that the UV measurements are not as accurate and repeatable as the visible spectrum measurements. In particular the UV beam shape was elliptical rather than circular as for the visible and IR incident beam. The UV beam was also affected by a small wedge in the rotating UV TAU optics causing a $\sim 0.3 \mathrm{~mm}$ beam wobble at the sample. The extinction ratio of the Wollaston prism in this wavelength region decreases from $\sim 1 \mathrm{e} 4$ to $\sim 1 \mathrm{e} 2$ and the detector sensitivity is falling rapidly over the $350-500 \mathrm{~nm}$ band. These shortcomings are easily overcome in a dedicated blue/UV beam line but for our broadband setup the blue/UV measurements generally have larger errors than the visible measurements as is evident in some of the figures. In separate experiments we varied the blue/UV and visible beam diameters and shapes in back to back PI and TAU measurements on the same sample and found that these gave consistent $( \pm 5 \%)$ results with the setup used for the main experiment.

\section{Measurements on biological materials}

4.1 Leaves - Light scattering from leaves has been the subject of many previous studies [see for example 26-34,37] but only a few $[26,27,30,34]$ address circular polarization and these have mainly been single wavelength $(633 \mathrm{~nm})$, multiple incidence angle measurements.

Leaf samples were examined from a variety of plants. We found that non- specular polarized back scattering from green leaf samples was similar from plants as dissimilar as bamboo, conifers, grasses, and deciduous trees although there were variations in detail as might be expected from colour and surface texture differences. The main features of the scattering from all the examined leaves are represented in three plants chosen for detailed presentation here, Arabidopsis thaliana (aka Thale Cress) [35]), English Oak (Quercus robur), and Ficus benjamina. A. thaliana is the genetically mapped 'standard plant' used in many biological experiments [36]. The leaf samples were tested within four hours of harvesting but only the $A$. thaliana leaves showed significant physical changes, mainly from dehydration, after up to eight hours from harvesting. Samples kept hydrated in sealed bags showed no significant changes after up to 48 hours. Sample to sample variability was larger than changes in measurements on a single sample over 1-2 hours.

Fig 2 shows the total scattering from three leaf samples relative to Spectralon. There were detailed differences between various samples depending on age, time since sampling, and colour variations but, the plot shows typically measured values. The red edge is very prominent and $A$. thaliana also shows a broad scattering peak at $\sim 600 \mathrm{~nm}$. The Q.robur. and F. benjamina total scattering may have strong features in the 500-560nm gap region of these measurements. 
Figs $3 a, 3 b, 3 c$ show the results of defined incident polarization (PI) measurements on $A$. thaliana, $Q$. robur, and $F$. benjamina. The magnitude of the scattered polarization fraction is shown in these figures with the sign of the component derived from the relative phase angle with respect to the PEM phase. At the scale of the graphs the right and left incident circular light scattering for all samples is not distinguishable by the amplitude of the scattered light so only the left circular scattering measurements are shown.

The $Q$. robur and $A$. thaliana measurements are quite similar except for the larger $Q(s)$ values, the (s) polarized backscattering from an (s) polarized input. The $F$. benjamina measurements from 560-650nm are relatively larger compared to the scattering peak than the other two leaves. None of the leaf samples show any significant scattering features from 350-500nm.

For the PI measurements the polarization of the scattered light is primarily the same as the incident polarization, i.e. $Q(s)$ and $V(L, R)$ are large as would be expected for scattering from a rough leaf surface [37], and there are significant and well-known spectral features due to the presence of chlorophyll $[14,15]$ scattering bodies. The scattered light shows a large range of polarization, depending on the incident wavelength. The scattering at $\sim 590 \mathrm{~nm}$ and $\sim 680 \mathrm{~nm}$ arises from antenna pigment absorption signatures and chlorophyll a respectively [14]. The characteristic 'vegetation red edge' in the polarized scattering is clearly shown with the backscattered light nearly completely polarized at the red edge and with small scattered polarization fraction above 700nm. This should be compared to the total scattered light, $\mathrm{m}_{11}$, measured relative to the Spectralon standard given in Fig 2. The total light scattering at longer wavelengths is large but the polarization fraction is small while the opposite is true at shorter wavelengths with smaller total light scattering that is highly polarized. The elevated polarization scattering $Q(s)$ is related to the surface roughness of the sample as additional Fresnel reflections would tend to increase the amount of (s) polarized light scattered into the analyzer.

Fig 4 shows the time-averaged unpolarized (TAU) measurements on all three plants. These measurements probe the scattering coefficients in the first column of the scattering matrix namely $m_{21}, m_{31}, m_{41}$. For the leaf samples $m_{21}>m_{41}>m_{31}$. A measure of the surface roughness is the level reached by the $m_{21}$ values at wavelengths longer than $\sim 750 \mathrm{~nm}$ as in the PI measurements. The $Q$. robur and $F$. benjamina leaves were shiny and waxy (glabrous) while $A$. thaliana leaves are microscopically rougher and covered with small hair-like filaments (trichomes) and the latter show larger values of polarized $Q(s)$ scattering at $>750 \mathrm{~nm}$ with approximately the same total scattering relative to Spectralon (see Fig 1).

4.2 Derived Scattering Coefficients - The PI measurements can be used to derive values for the individual scattering matrix coefficients. By taking differences of the $V(R)$ and $V(L)$ measurements when using right and left circular incidence values, $m_{44}$ and $m_{41}$ can be produced and similarly for $m_{21}, m_{24}, m_{31}$, and $m_{34}$. The TAU measurements give $m_{21}, m_{31}$ and $m_{41}$ directly for comparison. Further combinations lead to coefficients $m_{22}, m_{32}$, and $m_{42}$. Sample to sample variations and the difficulty of precisely illuminating the same area on repositioned samples mean that there are 
differences of $10-20 \%$ between the two techniques for different samples but the same spectral features are present.

Fig 5 shows the result of the calculations giving the magnitude of nine coefficients for A. thaliana. The magnitude of $\mathrm{m}_{14}$ was less than .003 for all wavelengths and is not shown. Figures $6 a$ and $6 b$ show the $m_{41}, m_{21}$ and $m_{42}, m_{24}$ coefficients for all three leaves. The scattering matrix coefficient values are remarkably similar in spite of the very different appearance of these samples.

Fig 7 shows a comparison of the two methods of defined polarization incidence (PI) and time averaged unpolarized incidence (TAU) measurements for $A$. thaliana. The agreement between the two methods is reasonable in the visible region but there are large differences in the blue/UV measurements. A total of four samples were used to compile these measurements, two for each technique and spectral region so considerable sample differences are expected.

The measurements on $Q$. robur and $F$. benjamina are very similar in appearance to A.thaliana with good agreement in the visible but with the blue/UV TAU measurements giving lower, 'flatter' spectral features compared to Figure 6a. This is at least partly attributable to the quality of the spinning waveplate in the UV TAU measurements versus the super-achromatic quarter wave plate used in the PI measurements. $Q$. robur and $F$. benjamina measurements taken back to back on single samples show similar behaviour between the two wavelength ranges and $\mathrm{PI} / \mathrm{TAU}$ methods and give scattering coefficients to within $\pm 2 \%$ in the visible region.

We observe some differences in $m_{21}, m_{31}$ and $m_{41}$ measurements depending on the orientation of the leaf surface with respect to the plane of incidence. The largest values are found for the three leaf samples oriented with the main leaf midrib parallel to the plane of incidence. In this orientation the values of $m_{21}, m_{31}$, and $m_{41}$ are $10 \%$ larger than the values in Fig 4 or 6 . This effect was not studied in detail but has been observed previously $[33,37]$.

The $Q$. robur results are consistent with previous measurements $[30,33]$ on oak leaves at $633 \mathrm{~nm}$ but the different incidence and scattering angles make a direct comparison difficult without further angular incidence measurements. Table 2 summarizes the leaf scattering coefficient PI measurements at $680 \mathrm{~nm}$ and $750 \mathrm{~nm}$ including the sign of the coefficients as derived from the phase angles of the polarization fraction measurements. 


\begin{tabular}{|l|l|l|l|l|l|l|l|l|l|l|}
\hline & $\lambda[\mathrm{nm}]$ & $\mathrm{m}_{21}$ & $\mathrm{~m}_{31}$ & $\mathrm{~m}_{41}$ & $\mathrm{~m}_{22}$ & $\mathrm{~m}_{44}$ & $\mathrm{~m}_{32}$ & $\mathrm{~m}_{42}$ & $\mathrm{~m}_{24}$ & $\mathrm{~m}_{34}$ \\
\hline A thal. & 680 & $.145 \pm$ & $.011 \pm$ & $.055 \pm$ & $.757 \pm$ & $-.326 \pm$ & $.079 \pm$ & $.299 \pm$ & $-.346 \pm$ & $-.212 \pm$ \\
& & .005 & .007 & .017 & .006 & .012 & .008 & .028 & .006 & .007 \\
\hline & 750 & $.013 \pm$ & $.007 \pm$ & $.013 \pm$ & $.232 \pm$ & $-.032 \pm$ & $.032 \pm$ & $.095 \pm$ & $-.060 \pm$ & $-.048 \pm$ \\
& & .002 & .001 & .002 & .003 & .003 & .002 & .058 & .003 & .008 \\
\hline Q.robur & 680 & $.138 \pm$ & $.017 \pm$ & $.050 \pm$ & $.818 \pm$ & $-.548 \pm$ & $.109 \pm$ & $.303 \pm$ & $-.441 \pm$ & $-.324 \pm$ \\
& & .002 & .002 & .019 & .003 & .041 & .003 & .026 & .003 & .028 \\
\hline & 750 & $.016 \pm$ & $.005 \pm$ & $.006 \pm$ & $.124 \pm$ & $-.046 \pm$ & $.023 \pm$ & $.048 \pm$ & $-.039 \pm$ & $-.025 \pm$ \\
& & .001 & .001 & .018 & .002 & .019 & .002 & .018 & .002 & .002 \\
\hline F. benj. & 680 & $.139 \pm$ & $.018 \pm$ & $.052 \pm$ & $.868 \pm$ & $-.610 \pm$ & $.107 \pm$ & $.337 \pm$ & $-.433 \pm$ & $-.337 \pm$ \\
& & .002 & .002 & .028 & .003 & .029 & .007 & .039 & .003 & .029 \\
\hline & 750 & $.014 \pm$ & $.006 \pm$ & $.005 \pm$ & $.125 \pm$ & $.047 \pm$ & $.021 \pm$ & $.048 \pm$ & $-.036 \pm$ & $-.026 \pm$ \\
& & .001 & .001 & .001 & .002 & .002 & .002 & .004 & .002 & .002 \\
\hline
\end{tabular}

Table 2. Calculated scattering coefficients for leaves from PI scattering data. The magnitude of the coefficient $\mathrm{m}_{14}$ is less than \pm .003 over the entire spectral range for the three leaf types.

\subsection{Measurements on Samples of Naturally-Occurring Bacteria}

To investigate microbial phototrophs, samples of different types of rock surface dwelling microbial communities were obtained. These include lichen and cyanobacterial epilithic (surface dwelling) communities. A diversity of lichens were obtained that naturally grow on the surface of volcanic rocks in Iceland were also examined. The samples were:

A Cyanobacteria (principally Gloeocapsa) biofilm on dolomite rock from the polar desert, Devon Island, Canadian High Arctic [38,39]

B Cyanobacteria (principally Lyngbya, Phormidium) biofilm on sandstone from Beer, Devon, UK [40]

C Cyanobacteria (Nostoc) Curled mat and sheets, Devon Island, Canadian High Arctic. [41]

D Cyanobacteria (Lichen) biofilm growing on volcanic basalt from the Isle of Skye, Scotland.

CC0709-1- 8 -- Lichen from Iceland on basalt, various species.

Pipwell - Green biofilm on limestone from Northhamptonshire, UK

Tile - Roof tile with black cyanobacteria deposits from Hertfordshire, UK

GypArc - Biofilm deposits on gypsum from the Canadian high arctic.

Atacama - Biofilm on crumbly limestone from the Atacama desert, Chile.

1980 - Lichen on pumice from the 1980 lava flow of the volcano Mt. Hekla, Iceland.

1913 - Lichen on pumice from the 1913 lava flow of Mt. Hekla, Iceland. 
These samples were challenging to illuminate consistently due to their irregular surfaces. Reasonably flat areas somewhat larger than the beam diameter were chosen and oriented at $45 \mathrm{deg}$ as for the leaf samples.

Most of the lichen samples were crustose, i.e. tightly bound to the surface of the substrate. Sample $\mathrm{C}$ is very different from the samples on rocks. The Nostoc mat resembles crumpled sheets of thin, semi-transparent brown paper when dry and is a brownish green slime when wet. All of the Nostoc data presented here is for samples that had been hydrated for at least two days. Figure 8 shows the total scattering seen for most of the samples listed previously. With the exception of sample B, the green cyanobacterial biofilm from Beer, Devon UK, the samples have lower total scattering than leaves. The chlorophyll 'red edge' is visible in most samples at $\sim 680 \mathrm{~nm}$ with antenna pigment signatures at $\sim 580$ and $620 \mathrm{~nm}$. Also present in some samples are significant scattering increases at wavelengths shorter than $380 \mathrm{~nm}$ and possible additional scattering at $\sim 800 \mathrm{~nm}$. The short wavelength scattering arises because of UV protecting pigments [41,42] particularly in the Atacama and GypArc samples but also in the lichen samples from Skye, Hekla, and Devon. The Nostoc sample from the Canadian high Arctic has a small total UV scattering in this measurement.

Figs 9 and 10 show the $Q(s)$ and $V(L) P I$ measurements, the largest scattering for these samples. As in the leaf samples a 'red edge' at $\sim 680 \mathrm{~nm}$ can be identified for the polarized scattering from most of these lichen and cyanobacteria samples but there are large differences in the polarized scattering at shorter wavelengths.

Nostoc shows a very broad double peak in both linear and circular polarized light scattering from $650-700 \mathrm{~nm}$ and a well defined peak at $\sim 400 \mathrm{~nm}$. The 1980 Hekla lichen sample shows a very broad peak from 480-550nm and a rapidly increasing scattering at $<390 \mathrm{~nm}$ for both linear and circular polarized incident light. There are numerous features in the sample spectra that suggest scattering by various pigments can be stronger than the principal photosynthetic pigment (Chlorophyll a). Sample A, Gloeocapsa, has the singular quality of having a larger V(L) scattering than $\mathrm{Q}(\mathrm{s})$. This is the only example of this behaviour amongst all the tested samples. This sample was only very thinly deposited on a substrate which had low scattering. Substrate scattering will be discussed in more detail in a later section.

All of the samples were measured with both the PI and TAU configuration and the agreement is good in the visible region as for the leaves. Since the PI technique offered better signal to noise, the majority of the results presented in this section will be PI measurements with derived coefficients except where noted. In general, the measurements show the same features, i.e. a 'red edge' and elevated scattering at $\sim 600 \mathrm{~nm}$ but the TAU spectral features are considerably less distinct. Some of the differences are due to variations in the sample placement between the two sets of measurements but the TAU measurements have consistently lower spectral contrast than the PI measurements. The small beam wobble at UV/blue wavelengths will also be a factor with these rough non-uniform samples as it will tend to average over a larger portion of the sample than for the visible measurements.

Figures 11,12 , and 13 show the $m_{41}$ and $m_{21}$ coefficients for the lichen and bacterial samples grouped by similarity of spectral features. In general the $m_{21}$ 
measurements are $\sim 3 x$ the $m_{41}$ values for most samples with the $D$ and Atacama samples being the exceptions at $\sim 2 x$ in the UV/blue portion of the spectrum. The Group 1 samples show polarized UV scattering that is continuing to rise strongly at $<350 \mathrm{~nm}$. The samples also show scattering peaks at $\sim 450-500 \mathrm{~nm}$ with the 1980 sample having a very pronounced broad scattering peak at $\sim 500 \mathrm{~nm}$. In the visible spectrum these samples show only a weak scattering peak at $\sim 680 \mathrm{~nm}$. All of these samples have large numbers of black fruiting bodies with the Tile sample being uniformly black. The gap between $500-560 \mathrm{~nm}$ is unfortunate as there are hints of further scattering structures in this region.

In Group 2 sample D has two peaks at $\sim 370 \mathrm{~nm}$ and $\sim 450 \mathrm{~nm}$ and only a small perturbation at $\sim 680 \mathrm{~nm}$. Sample A shows significant differences between the $m_{21}$ and $\mathrm{m}_{41}$ spectral features at $\sim 400 \mathrm{~nm}, \sim 440 \mathrm{~nm}$, and $\sim 640 \mathrm{~nm}$. Again, the gap at 500 $560 \mathrm{~nm}$ does not allow the resolution of further scattering peaks that might be present. These two samples have a typical lichen appearance, i.e. grey with black fruiting bodies covering the rock surface but are quite thin compared to the Group 1 or 3 samples.

The Group 3 samples have similar characteristics in the blue/UV and visible having a peak in the $\mathrm{m}_{21}$ and $\mathrm{m}_{41}$ coefficients at $\sim 400 \mathrm{~nm}$ which might further resolved into components at $\sim 370 \mathrm{~nm}$ and $\sim 440 \mathrm{~nm}$. Most of these samples exhibit broad scattering in the visible between $\sim 610 \mathrm{~nm}$ and $\sim 670 \mathrm{~nm}$. There are small spectral differences in the $m_{21}$ versus $m_{41}$ scattering measurements as can be seen in the peak values of the Nostoc sample which are similar to that of the leaves in Fig 6a. The Nostoc mat and sample B are physically quite different from the other samples as previously noted. The Atacama, GypArc, and Nostoc samples come from regions with extreme climatic conditions.

\subsection{Substrate Scattering}

Background scattering from the various rocky substrates is of concern if there are significant spectral features that could compete with the biological scattering processes. A number of measurements were performed on the bare substrate rocks and two types of nonorganic materials that have spectral features. Fig 14 shows the total scattering relative to Spectralon for several of the rock substrates, iron oxide powder ( $5 \mu \mathrm{m}$, Sigma-Aldrich 31005), and a red solvent based paint (Ford Sunlight Red, Halfords). The red paint and iron oxide have low total scattering at $550 \mathrm{~nm}$ which rises steeply to $650-700 \mathrm{~nm}$. The curve labelled -8 fungus was measured for the grey lichen fungal layer on a sample that had no visible darker fruiting bodies. It nevertheless shows a small total scattering feature at $\sim 680 \mathrm{~nm}$ and is distinguishable from the rock substrate CC0709-8.

Fig 15 shows the TAU $m_{41}$ measurements on the substrates corresponding to the samples. Note that the scattering coefficients are smaller than the coefficients of the lichen covered samples and lack any distinctive spectral features. In general the substrates show only a slow fall in scattering with increasing wavelength. The $m_{21}$ measurements for the substrates were $\sim 4 \mathrm{x}$ larger but had the same spectral shapes. The curve labelled 8-fungus $m_{41}$ is the same sample as in the previous figure measured where no darker fruiting bodies are visible and shows a small feature at $\sim 680 \mathrm{~nm}$. The sample labelled 5 -fungus $\mathrm{m}_{41}$ is a different uniformly white lichen 
sample and shows no significant features. The smoother samples showed coefficients with low values while the roughest samples have higher scattering.

\section{Analysis and Discussion}

Although there have been many published studies on the spectropolarimetry of leaves and other biological materials the parameter space is very large and the overlaps between studies is small. The published papers closest to that presented here on leaves are those of Pospergilis [27], Krishnan, et al [34], and Savenkov, et al $[30,33]$. Pospergilis reported Stokes measurements on a green leaf, as a function of wavelength, with a fixed scattering geometry, similar to the measurements reported here. The general form and magnitudes of the equivalent $Q, U$, and $V$ measurements are consistent with our detailed measurements. In several studies Savenkov reported Mueller matrix coefficients at a fixed wavelength $(633 \mathrm{~nm})$ with varying incidence and backscattering angles for wheat leaves [26] and English oak leaves [33]. Krishnan reported Mueller matrix coefficients for yew and maple leaves at $633 \mathrm{~nm}$ with a fixed incidence angle of $40 \mathrm{deg}$ measuring the reflected light from the leaf surface.

The Savenkov Mueller scattering coefficients are consistent with the leaf measurements here at $630 \mathrm{~nm}$ although the exact incidence and scattering angles are not the same. The measurements have slowly varying coefficients with (Fresnel) backscattering angle. The Krishnan measurements are principally forward scattering and the coefficients differ in magnitude and sign to the Savenkov measurements and the present experiment. We conclude that these differences are due to the dominance of surface reflections in their configuration.

Direct comparison with the scattering measurements on cultured photoactive bacteria, and maple leaves of Sparks et al $[5,6]$ is difficult because of the differing illumination geometries. In Sparks et al unpolarized illumination arrives at the sample from all angles and the scattering from the sample is observed normal to the surface. The TAU measurements on leaves in this paper are the closest to the maple leaf measurements but are at fixed incidence and backscattering angles. Further study is needed on the angular dependence of polarized scattering near the chlorophyll red edge to bring all techniques into a consistent framework.

A general observation from this survey of the spectral dependence of polarized backscattering from biological materials is that strongly polarized scattering occurs near regions where selective absorption by the material is present (the Cotton effect), as has been observed previously. The red edge from chlorophyll absorption gives a very characteristic shape to the linearly polarized scattering from green leaves. The circularly polarized scattering is in part due to the chiral nature of the chlorophyll absorption. The circular scattering in the PI configuration is dominated by the incident polarization and the small right/left differences require careful treatment of the amplitude and phase information in the PEM modulated signals to extract scattering coefficient information that is consistent with unpolarized circular scattering. The negative sign of $\mathrm{V}(\mathrm{L})$ and the positive sign of the scattering coefficient $m_{41}$ is indicative of an overall left circular backscattering from both leaves and the cyanobacteria/lichen samples in our measurement configuration. 
The presence of other absorption processes related to light harvesting via phycobiliproteins and carotenoids also gives rise to polarized scattering spectral features which can also exhibit chirality $[5,6]$. Some cyanobacteria samples, Nostoc and others, show high polarization fractions and low total scattering at short wavelengths, red edge behaviour in total and polarized backscattering, and higher total scattering with low polarization fractions at longer wavelengths similar to leaves. Groups 1, 2, and 3 exhibit a variety of scattering processes but there are several common features that could be used as biomarkers.

Common to both green leaves and the lichen and cyanobacterial samples we measured here are increases in the circular and linear polarization scattering coefficients at the chlorophyll red edge at $\sim 680 \mathrm{~nm}$ and broad peaks at shorter wavelengths from antenna pigments assisting the photosynthetic processes. At much shorter wavelengths polarized scattering peaks appear due to absorption by UV protecting pigments and in some cases these are the largest observed. The enhanced scattering near absorption peaks can be seen in the total scattered light from the samples and the polarized scattering can be easily separated from background substrate scattering. Polarized scattering, arising from multiple light scattering in the substrates, may contribute to an overall background but is not likely to introduce any spectral features that could emulate scattering from biofilms if there is significant absorption near the surface.

Kiang et al $[43,44]$ have published an extensive review of the spectroscopic properties of the vegetation red edge and extrapolated to a number of extrasolar examples that could lead to useful biosignature identification techniques. If in addition the polarization properties that are observed near the red edge and in other spectroscopic positions are added to the spectroscopic analysis, the robustness of the techniques may be significantly enhanced. The detection of circular polarized scattering near the red edge might prove to be particularly interesting for the identification of extrasolar photosynthesis. Ultimately the number of collected photons will determine the extent of diagnostic techniques that are available. Polarization techniques would not necessarily detract from overall detection efficiencies if they were included in the basic design of future instruments.

If the incident light is so strongly absorbed by the material that the penetration depth is small and internally backscattered light reaching the surface is correspondingly small then only surface scattering would be seen. We suspect this is the reason for the very small spectral variation in the two featureless lichen samples that would have their chlorophyll deeply buried. This raises an important point for the detection of exobiological photosynthesis: the absorption process cannot be so efficient as to eliminate the possibility of detecting the scattered light as pointed out by Kiang et al. Very strong absorption at short wavelengths (for example for UV protection) or at longer wavelengths to enhance the photosynthesis processes may result in quite different polarized scattered signatures than we might expect from plants and bacteria evolved under our G2V sun.

\section{Conclusions and Recommendations}

We have measured spectral dependencies of Stokes backscattering matrix coefficients for leaves and biofilms in the laboratory including the infrequently 
addressed circular scattering components. The elastic backscattering coefficients can be large ( 0.5 , i.e, the scattered light is more than $50 \%$ polarized) for many biological and plant samples near the chlorophyll red edge and where strong absorption is present. The spectral dependence of the circular backscattering, particularly the $m_{41}$ coefficient, is a promising feature that could be used to strongly discriminate the signatures of phototrophic organisms using light harvesting processes that include chiral molecules. The magnitude of $\mathrm{m}_{41}, \sim .05$ for leaves near the red edge, $\sim 0.005$ at longer wavelengths, means that unpolarized light backscattered from leaves could be as much as $5 \%$ circular polarized compared to longer wavelengths. For near-specular scattering the polarization fractions near the red edge could be even higher according to previous angular dependence studies at $633 \mathrm{~nm}$ wavelengths.

The smaller biofilm $\mathrm{m}_{41}$ values, .02 have less contrast at longer wavelengths and may present challenges as a discriminant particularly in the presence of protective mechanisms or high absorption to enhance light collection. The statistics of the $m_{41}$ PI measurements ( $n=$ five sample measurements in the survey reported here) would benefit from a larger sample measurement set.

Further work is needed on the angular dependence of linear and circular polarized light scattering from chlorophyll containing biological material and this might best be accomplished by investigating the scattering at multiple incidence angles with measurements at $\sim 680 \mathrm{~nm}$ and $\sim 750 \mathrm{~nm}$. The papers of Kiang et al $[43,44]$ give an estimation of how these particular wavelengths might vary with convergent evolution under different extrasolar conditions and therefore might then be incorporated into the design of instruments for the detection of extrasolar photosythesis.

\section{Acknowledgements}

This work has been supported by a grant from the Leverhulme Trust (Grant number: F/00 281/F) and by funding from the University of Hertfordshire Science Research Infrastructure Fund (SRIF).

\section{References}

1] Gledhill TM, Sparks WB, Ulanowski Z, Hough JH, DasSarma S. Astro-biological signatures - prospects for the detection of non-terrestrial biological material. In: Optics of Biological Particles. ed: Hoekstra A, Videen G, Maltsev V. Dordrecht: Springer; 2007:193-211.

2] Schmid HM, Beuzit J-L, Feldt M, Gisler D, Gratton R, Henning Th, Joos F, Kasper M, Lenzen R, Mouillet D, Moutou C, Quirrenbach A, Stam DM, Thalmann C, Tinbergen J, Verinaud C, Waters R, Wolstencroft R. Search and investigation of extra-solar planets with polarimetry. Direct Imaging of Exoplanets: Science \& Techniques Proceedings IAU Colloquium No. 200; 2005: 165-170

3] Seager S, Turner EL, Schafer J, Ford EB. Vegetation's Red Edge: A Possible Spectroscopic Biosignature Of Extraterrestrial Plants. Astrobiology 2005; 5(3): 372-390 
4] Raven JA, Cockell CS. Influence on Photosynthesis of Starlight, Moonlight, Planetlight, and Light Pollution (Reflections on Photosynthetically Active Radiation in the Universe). Astrobiology 2006; 6(4): 668-675

5] Sparks WB, Hough J, Germer TA, Chen F, DasSarma S, DasSarma P, Robb FT, Manset N, Kolokolova L, Reid N, Macchetto FD, Martin W. Detection of circular polarization in light scattered from photosynthetic microbes. Proceedings of The National Academy of Sciences of the United States of America 2009; 106(26): 10871-10871

6] Sparks WB, Hough JH, Kolokolova L, Germer TA, Chen F, DasSarma S, DasSarma P, Robb FT, Manset N, Reid IN, Macchetto FD, Martin W. Circular polarization in scattered light as a possible biomarker. Journal of Quantitative Spectroscopy and Radiative Transfer 2009; 110(14-16): 1771-1779

7] Arnold L, Gillet $S$, Lardière $O$, Riaud $P$, Schneider J. A test for the search for life on extrasolar planets-Looking for the terrestrial vegetation signature in the Earthshine spectrum. Astronomy and Astrophysics 2002; 392: 231-237

8] Hamdani S, Arnold L, Foellmi C, Berthier J, Billeres M, Briot D, François P, Riaud $P$, Schneider J. Biomarkers in disk-averaged near-UV to near-IR Earth spectra using Earthshine observations. Astronomy and Astrophysics 2006; 460: 617-624

9] Montanes-Rodriguez P, Palle E, Goode PR, Hickey J, Koonin SE. Globally Integrated Measurements Of The Earth's Visible Spectral Albedo. The Astrophysical Journal 2005; 629:1175-1182

10] Woolf NJ, Smith PS, Traub WA, Jucks KW. The Spectrum Of Earthshine: A Pale Blue Dot Observed From The Ground. The Astrophysical Journal 2002; 574:430_ 433

12] Seager S, Turner EL, Schafer J, Ford EB. Vegetation's Red Edge: A Possible Spectroscopic Biosignature Of Extraterrestrial Plants. Astrobiology 2005; 5(3): 372390

11] Vazquez M, Montanes-Rodriguez $P$, Palle E. The Earth As An Object Of Astrophysical Interest In The Search For Extrasolar Planets. Lecture Notes and Essays in Astrophysics 2006; 2: 49-70

13] Zarco-Tejada PJ, Miller JR. Land cover mapping at BOREAS using red edge spectral parameters from CASI imagery. Journal of Geophysical Research 1999; 104(D22): 27921-27933

14] Bacon KE. Chlorophyll-Protein Complexes Part III: Optical Rotary Dispersion of Chlorophyll-Containing Particles from Green Plants and Photosynthetic Bacteria. in Vernon LP, Seely GR (eds). The Chlorophylls. New York: Academic Press;1966:

Chapter 13 
15] Houssier C, Sauer K. Cirular Dichroism and Magnetic Circular Dichroism of the Chlorophyll and Protochlorophyll Pigments. Journal of the American Chemical Society 1970; 92(4): 779-791

16] Vidal-Madjar A, Desert J-M, Lecavelier des Etangs A, Hebrard G, Ballester GE, Ehrenreich D, Ferlet R, McConnell JC, Mayor M, Parkinson CD. Detection Of Oxygen And Carbon In The Hydrodynamically Escaping Atmosphere Of The Extrasolar Planet Hd 209458b. The Astrophysical Journal 2004; 604:L69-L72

17] Hough JH, Lucas PW, Bailey JA, Tamura M, Hirst E, Harrison D, BartholomewBiggs M. PlanetPol: A Very High Sensitivity Polarimeter. Publications of the Astronomical Society of the Pacific 2006; 118:1302-1318

18] Schmid HM, Gisler D, Joos F, Povel HP, Stenflo JO, Feldt M, Lenzen, W. Brandner R, Tinbergen J, Quirrenbach A, Stuik R, Gratton R, Turatto M, Neuhäuser R. ZIMPOL/CHEOPS: a Polarimetric Imager for the Direct Detection of Extra-solar Planets. Astronomical Society of the Pacific ASP Conference Series 2005; 343:8991

19] Garab G, Leegood RC, Walker DA, Sutherland JC, Hind G. Reversible changes in macroorganization of the light-harvesting chlorophyll a/b pigment-protein complex detected by circular dichroism. Biochemistry 1988; 27(7):2430-2434

20] Lewis JW, Goldbeck RA, Kliger DS, Xie X, Dunn RC, Simon JD. Time-resolved circular dichroism spectroscopy: experiment, theory, and applications to biological systems. Journal of Physical Chemistry 1992; 96 (13): 5243-5254

21] Tinbergen J. Astronomical Polarimetry. New York: Cambridge University Press; 1996. Chapter 4

22] Bickel WS, Bailey WM. Stokes vectors, Mueller matrices, and polarized scattered light. American Journal of Physics1985; 53(5): 468-478

23] Anderson R. Measurement of Mueller matrices. Applied Optics1992; 31(1):11-13

24] Compain E, Poirier S, Drevillon B. General and self-consistent method for the calibration of polarization modulators, polarimeters, and Mueller-matrix ellipsometers. Applied Optics1999; 38(16): 3490-3502

25] www.labsphere.com Reflectance standards.

26] Savenkov SN, Mishchenko LT, Muttiah RS, Oberemok YA, Mishchenko IA. Mueller polarimetry of virus-infected and healthy wheat under field and microgravity conditions. Journal of Quantitative Spectroscopy and Radiative Transfer 2004; 88: 327-343

27] Pospergelis MM. Spectroscopic Measurements of the Four Stokes Parameters for Light Scattered by Natural Objects. Soviet Physics - Astronomy 1969; 12(6): 973976 
28] Atrashevskii YI, Sikorskii AV, Sikorskii VV, Stermakh GF. The Reflection And Scattering Of Light By A Plant Leaf. Journal of Applied Spectroscopy 1999; 66(1): 105-114

29] Grant L, Daughtry CST, Vanderbilt VC. Polarized and specular reflectance variation with leaf surface features. Physiologia Plantarum 1993; 88: 1-9

30] Savenkov SN, Muttiah RS. Inverse Polarimetry and Light Scattering From Leaves. in Videen G et al (eds), Photopolarimetry in Remote Sensing, NATO Science Series II; 161: 243-264; Netherlands: Springer; 2004

31] Voshchula IV, Zhumar AY, Tsaryuk OV. Elliptical Polarization of Laser Light Reflected from Plant Leaves and Characteristics of the Leaf Cuticle. Biophysics 2007; 52(4): 418-422

32] Tsaruk AV, Zaitseva VA, Zhumar AY. Ellipticity of He-Ne laser radiation reflected by leaves near brewster angle. Proceedings SPIE 2004; 5566: 11-14

33] Savenkov SN, Muttiah RS, Oberemok YA. Transmitted and reflected scattering matrices from an English oak leaf. Applied Optics 2003; 42(24): 4955-4962

34] Krishnan S, Nordine PC. Mueller-matrix ellipsometry using the division-ofamplitude photopolarimeter: a study of depolarization effects. Applied Optics 1994; 33(19): 4184-4192

35] Arabidopsis genotype NASC N1092, see http://arabidopsis.info/

36] see for example Kao FJ, Wang YM, Chen JC, Cheng PC, Chen RW, Lin BL. Micro-spectroscopy of chloroplasts in protoplasts from Arabidopsis thaliana under single- and multi-photon excitation. Journal of Luminescence 2002; 98: 107-114

37] Ma Q, Ishimaru A, Phu P, Kuga Y. Transmission, Reflection, and Depolarization of an Optical Wave for a Single Leaf. IEEE Transactions on Geoscience and Remote Sensing 1990; 28(5): 865-871

38] Cockell CS, Stokes MD. Widespread colonization by polar hypoliths. Nature 2004; $431: 414$

39] Cockell CS, Stokes MD. Hypolithic colonization of opaque rocks in the Arctic and Antarctic Polar Desert. Arctic, Antarctic and Alpine Research 2006; 38: 335-342

40] Olsson-Francis K, de la Torre R, Cockell, CS. Isolation of novel extreme-tolerant cyanobacteria from a rock-dwelling coastal environment using exposure to low Earth orbit. Applied and Environmental Microbiology 2010 (in press)

41] Cockell CS, Rettberg P, Horneck G, Scherer K, Stokes DM. Measurements of microbial protection from ultraviolet radiation in polar terrestrial microhabitats. Polar Biology 2003; 26: 62-69 
42] Cockell CS, Knowland J. Ultraviolet radiation screening compounds. Biological Reviews 1999; 74: 311-345.

43] Kiang NY, Siefert J, Govindjee, Blankenship RE. Spectral Signatures of Photosynthesis. I. Review of Earth Organisms. Astrobiology 2007; 7(1): 222-250

44] Kiang NY, Segura A, Tinetti G, Govindjee, Blankenship RE, Cohen M, Siefert J, Crisp D, Meadows VS. Spectral Signatures of Photosynthesis. II. Coevolution with Other Stars and the Atmosphere on Extrasolar Worlds. Astrobiology 2007; 7(1): 252274 